\title{
Food knowledge and IMD score of Year 6 children participating in the CHANGE! Project
}

\author{
G. L. Warburton ${ }^{1}$, E. Mahon ${ }^{1}$, I. G. Davies ${ }^{1}$, R. Gobbi ${ }^{3}$, K. Mackintosh ${ }^{4}$, S. J. Fairclough ${ }^{2}$ \\ and J. C. Abayomi ${ }^{1}$ \\ ${ }^{1}$ Faculty of Education, Community and Leisure, Liverpool John Moores University, IM Marsh Campus, Barkhill Road, \\ Liverpool L17 6BD, ${ }^{2}$ Research Institute for Sports and Exercise Sciences, Liverpool John Moores University, \\ Liverpool, L3 3AF, ${ }^{3}$ Faculty of Science and Social Science, Liverpool Hope University, Liverpool L16 $9 J D$ and \\ ${ }^{4}$ Applied Sports Technology Exercise and Medicine Research Centre (A-STEM), College of Engineering, Swansea \\ University, Swansea SA2 8 PP
}

'Indices of Multiple Deprivation' (IMD) are used as a measurement for deprivation within a community, city or local authority ${ }^{(1)}$. The area with the most deprivation, will have a rank of 1 or 'first ${ }^{6(1)}$. Whilst previous studies have investigated the associations between deprivation and unhealthy diets ${ }^{(2)}$, and with childhood overweight and obesity ${ }^{(3)}$, the connection with knowledge of food and nutrition has received less attention. The CHANGE! Project (Children's Health, Activity, and Nutrition: Get Educated!), involving Year 6 children ( $n=290$ total; $n=138$ intervention; $n=152$ control) in Wigan, aimed to promote the benefits of healthy eating and physical activity, by making small behavioural changes to lifestyle. A 20 -week teaching programme, adapted from the Planet Health ${ }^{(4,5)}$ resources, was delivered by teachers in the intervention schools after the baseline (BL) data collection was completed.

Residential postcode information was collected for each participant at BL and post-intervention (PI). The participants' individual postcodes were converted to IMD scores using GeoConvert ${ }^{(6)}$. These scores were ranked and stratified into quartiles. The participants also completed a validated food knowledge questionnaire ${ }^{(7)}$ at each data collection point. A total food knowledge score was calculated for each participant at each point, with a possible maximum score of 59. At BL, there was a significant difference between the mean total food knowledge scores of those participants from the areas of lowest deprivation in Wigan $($ score $=30 \cdot 41)$ who scored higher than those from the areas of highest deprivation $($ score $=25 \cdot 38)(p=0 \cdot 024)$.

\begin{tabular}{llrll}
\hline & \multicolumn{3}{l}{ Data collection points } \\
\cline { 2 - 5 } & & \multicolumn{3}{l}{ PI } \\
mean & \\
& BL mean score & S.D. & score & S.D. \\
\hline $\mathbf{1 . 0 0}$ (lowest level of deprivation) & $30 \cdot 41^{*}$ & $8 \cdot 67$ & $31 \cdot 89$ & $9 \cdot 96$ \\
$\mathbf{2 . 0 0}$ & $28 \cdot 44^{\dagger}$ & $9 \cdot 81$ & $31 \cdot 50^{\dagger}$ & $9 \cdot 82$ \\
$3 \cdot 00$ & $28 \cdot 53$ & $10 \cdot 37$ & $30 \cdot 08$ & $8 \cdot 50$ \\
$4 \cdot 00$ (highest level of deprivation) & $25 \cdot 38^{* \dagger \dagger}$ & $9 \cdot 54$ & $29 \cdot 04^{\dagger \dagger}$ & $8 \cdot 53$ \\
\hline$*(1 \cdot 00>4 \cdot 00 ; p=0 \cdot 024),{ }^{\dagger}(p=0 \cdot 001),{ }^{\dagger \dagger}(p=0 \cdot 001)$. & & &
\end{tabular}

At PI, there was an increase in mean total food knowledge scores for all IMD score quartiles but there were no significant differences between the groups $(p=0 \cdot 702)$. The mean scores significantly increased however between BL and PI for groups $2 \cdot 00(p=0 \cdot 001)$ and $4.00(p=0.001)$. The mean scores at PI may potentially have been influenced by exposure to the questionnaire at BL. The increases in scores for the participants from the areas of highest deprivation were potentially influenced by the high percentage of intervention children in this group, who had received the teaching programme. Additionally, all mean scores, at BL and PI, were significantly different to the possible maximum score of $59(p=0 \cdot 000)$. These results demonstrate that deprivation does not necessarily preclude individuals from knowledge about food or nutrition. It is possible that there was a positive effect of the CHANGE! teaching programme, on the fourth quartile of participants.

1. Department for Communities and Local Government. The English Indices of Deprivation 2010.: Crown Copyright; 2011 [13th August 2013]. Available from: https://www.gov.uk/government/organisations/department-for-communities-and-local-government/series/english-indices-ofdeprivation.

2. Donkin AJ, Dowler EA, Stevenson SJ, Turner SA (2000) Mapping access to food in a deprived area: the development of price and availability indices. Public Health Nutrition 3(1), 31-8.

3. Cetateanu A, Jones A. (2014) Understanding the relationship between food environments, deprivation and childhood overweight and obesity: Evidence from a cross sectional England-wide study. Health and Place 27, 68-76.

4. Cheung L, Dart H, Kalin S, Gortmaker SL (2007) editors. Eat Well and Keep Moving. 2nd ed. Champaign, IL: Human Kinetics.

5. Carter J, Wiecha J, Peterson KE, Nobrega S, Gortmaker SL (2007) editors. Planet Health. 2nd ed. Champaign, IL: Human Kinetics.

6. UK Data Service Census Support. GeoConvert. 2012-2013 [August 2011]. Available from: http://geoconvert.mimas.ac.uk/.

7. Anderson AS, Bell A, Adamson AJ, Moynihan P 2002A Questionnaire Assessment of Nutrition Knowledge - Validity and Reliability Issues. Public Health Nutr.5(3), 497-503. 